\title{
An Overview of 3D Body Scanning Applications in Thailand
}

\author{
Supiya CHAROENSIRIWATH ${ }^{*}$ and Chularat TANPRASERT ${ }^{*}$ \\ Knowledge Elicitation and Archiving Laboratory (KEA) \\ National Electronics and Computer Technology Center (NECTEC) \\ 112 Thailand Science Park, Phahon Yothin Road \\ Klong 1, Klong Luang, Pathumthani 12120, THAILAND
}

\begin{abstract}
3D body scanning technology was first introduced in Thailand during the Bangkok Fashion Week in 2006. The event was organized by the Thai National Sizing Survey project, known as SizeThailand, to allow the people in the apparel and fashion industry to familiarize themselves with the latest method for accurately measuring body size and to make the general public aware of this new technology prior to the official launch of the project. SizeThailand was then launched in 2007 and successfully completed in 2008 with 13,442 subjects taking part. It followed the same procedures and methodology as SizeUK and SizeUSA which made Thailand the first country in Asia Pacific to have carried out an anthropometric survey using the 3D body scanning technology.
\end{abstract}

The results of the survey were publicly announced and most importantly, the country's first standard sizing scheme was developed using a novel Artificial Intelligence technique to analyse the body measurements obtained from the 3D body scanner. Since then, the 3D body scanning technology continued to attract a high level of media interest in Thailand. As a result, made-to-measure suits and uniforms trials have been conducted with 2 tailors and a clothing retailer. While the results were shown to be satisfactory, there is still room for improvement. Another important 3D body scanning application, arose from the survey results which found that over thirty percent of Thai adults were obese, was the SizeThailand e-Health: online obesity diagnosis and monitoring tool. The system allows the subjects taking part to monitor their body shape, clinical data, body composition monitor results and overall health online. In addition, the subjects periodically receive personal obesity-related advice and eating recommendations from doctors and nutritionists. A trial of this application has been running for the past 2 years with nearly 400 subjects taking part. This paper discusses these 3D body scanning applications in detail.

Keywords: SizeThailand, Made-to-Measure, e-Health, health monitoring

\section{Introduction}

The first national sizing survey in Thailand, known as SizeThailand, was initiated in 2006 by NECTEC to survey the body size and shape of the Thai population. SizeThailand was jointly funded by the government, three clothing retailers and an automotive company. In order to maximize the impact of the national sizing survey, a steering committee was formed to help in identifying the required body measurements and determining the statistical sample of subjects to be recruited. The committee comprised representatives from the following sectors:

- Government: Thai Industrial Standards Institute, National Statistical Office, the Federation of Thai industries and the National Science and Technology Development Agency

- Textiles organizations: Thai Tailors Association, Thailand Textile Institute and Thai Garment Development Foundation

- Clothing retailers: Thanulux, Thai Wacoal and Tesco Lotus

- Academia: Department of Textile Science at Kasetsart University, Nutrition Division at Ramathibodi hospital, IT and Industry Pattern Development Center

- Automotive: Toyota Motors Asia Pacific Engineering \& Manufacturing 
As it was not possible to collect data from everyone in the country, a statistically significant sample of subjects needed to be recruited. Quota sampling was used for selecting participants and the recruitment criteria were gender (male and female), age (5 age groups: 16-25, 26-35, 36-45, 46-59, 60+) and region (5 geographical regions containing 14 data collection locations). Therefore, throughout the data collection period, information on the subjects such as age, region of residence, socio-economic data, as well as size and body mass index data had to be continuously monitored in real-time so that those eligible would be chosen to take part. A real-time data monitoring and management system was developed specifically for this task and full details of the system can be found in [1].

SizeThailand took 14 months to collect data in 5 regions of Thailand (Bangkok, Central, North, North East and South). One TC ${ }^{2}$, S 3D body surface scanner was used and moved consecutively to 14 different locations. As a result, a national anthropometrics database of the Thai population was constructed. In total, there were 13,442 subjects, aged 16 and over from 74 out of 76 provinces, taking part in the project. The information contained in the database includes over 140 body measurements, 3D body shape, together with demographic, health, eating and shopping lifestyle data.

As the key project deliverable, by employing a novel Artificial Intelligence technique, a national standard sizing system was produced and publicly announced. Prior to this, there was no proper size designation standard (size charts) to determine the body sizes of the Thai people. Because of this, different brands of clothes in Thailand had various different sizes, making it difficult for people to find clothes that fitted well. As this was the country's first body size charts, the project had attracted high media attention and this led to our on-going research on made-to-measure which is described in more detail in section 3.

The SizeThailand data was also analysed through various statistical methods. One important finding was that over $30 \%$ of the Thai population is currently overweight or obese. This number was increased from 27\% in 2003 when the Department of Health conducted their diet and nutrition survey [2]. This showed that Thailand was one of the countries that have been experiencing an obesity epidemic. In an attempt to help reduce obesity, an online obesity diagnosis and monitoring tool was developed to make use of the 3D body scanning technology, allowing people to keep in touch with doctors and nutritionists. At the same time, doctors could also monitor their patients and screen for obesity at earlier stages. An earlier version of the system could be found in [3]. The latest version of the system, with improved capabilities, is discussed here in section 3 .

This paper is organized as follows: section 2 outlines related works and section 3 presents 2 case studies describing the ways in which 3D body scanning technology has been employed in the apparel and healthcare industries in Thailand. Finally, section 4 concludes.

\section{Background and Related Works}

\subsection{D Body Scanning in Apparel Industry}

Since 3D body scanners have been developed primarily for measuring a person's body size, it is not surprising to see that most 3D body scanning applications have been applied to the apparel industry [4]. SizeUK and SizeUSA pioneered the use of $3 \mathrm{D}$ whole body scanners for conducting national sizing surveys, and inspired similar surveys in France, Korea, Mexico, Brazil and Australia [5]. These national surveys were jointly funded by the government and the major national clothing \& textiles companies; interested in measurement data for better fitting garments.

Made-to-Measure clothing is another area that has benefited greatly from this technology. Examples of this include MTM Jeans at Selfridges in London by Bodymetrics [6] and Brooks Brothers Digital Tailoring [7]. Although, the selling point for these products seems to be the 'perfect fit', there are still a few things which can be improved to attract more customers. First, the price of these products is still too high for ordinary people. Second, in most cases, there is still a need for some further measurements to be taken by hand. Lastly, the products still take quite a long time to be manufactured; the usual turnaround time is between 2-4 weeks. This seems too long when it is now possible to get custom made clothes done within 24 hours in countries like Thailand or Hong Kong. 


\subsubsection{Body Scanning in Healthcare}

Outside the apparel industry, 3D body scanning has also been very popular with a myriad of applications across various industries, one of which is healthcare [5]. Here, 3D body scanners are used to track changes in body size and shape over time, mostly for diet and fitness programs and basic healthcare research. It is not difficult to see that customers, dieticians and/or fitness instructors can certainly benefit from a tool which offers visualization and statistical analysis of changes in body shape, size and body mass index (BMI), resulting from exercise or nutritional programs. Currently available tools include "My ImageTwin Weight Loss Simulation" by $\mathrm{TC}^{2}$ [8] which allows users to simulate their weight loss by entering their own body measurements to create a body shape silhouette and "Digital Body" where customers send in their 3D scans and a report is produced showing how the body measurements, BMI, together with waist-hip ratios, have changed over time [9].

\section{Case Studies: 3D Body Scanning Applications in Thailand}

In Thailand, there are currently two main research projects being conducted using the 3D Body scanning technology. Both are carried out by NECTEC. Their details are described as follows:

\subsection{Made-To-Measure Clothing}

Since the 3D body scanning technology has only been around in Thailand for less than 5 years, it is understandable that most local tailors are still getting acquainted with the new concept of measuring a person's body size and shape using something else other than hands. For Made-To-Measure using 3D body scanning to work in Thailand, the challenge is actually to convince the tailors to accept that this technology is here to help rather than to replace them. Therefore, the objective of this research is to come up with a new MTM process that works best for Thai tailors. So far, a number of suits and uniforms trials have been conducted with Made-to-Measure tailors, manufacturers and fashion students. Due to different kinds of tailoring involved, the way in which the trial was conducted varied slightly depending on the tailor type. In general, the trials followed the process shown in fig. 1.

First, the body measurements required by each tailor has to be obtained. Then, the measurements extracted from the 3D body scanner need to be fine-tuned, making them as close as possible to the manual measurements by that particular tailor. Once the fine-tuning is completed, the subjects taking part in the trial are scanned and measurements are extracted. An automatic tool has been developed to remove noise, fill holes and manipulate the $3 D$ scans so that they can be imported directly into a 3D pattern making software. For the trials, OptiTex was used in digitizing paper patterns and for virtual try-on. Suits patterns are adjusted until they are ready to be manufactured. The suits are then made and the subjects try them on. This follows by a fit evaluation which is carried out by a group of experts and lastly, final manual adjustments are made to the suits until they are well fitted to the subject's body. The success of a trial is measured by the percentage of the suits being well fitted, meaning that little or no alterations are required after the suits are made. Although results from previous trials were shown to be satisfactory, there is still room for improvement and more trials with different tailors and manufacturers need to be conducted. Table 1 shows summarised results of a Made-To-Measure uniform trial where 10 subjects (5 male and 5 female) took part and the uniform consists of a two-piece suit (jacket and trousers) for men and a three-piece suit (jacket, trousers and skirt) for women.

Table 1: Uniform Trial Results

\begin{tabular}{|c|c|c|c|c|}
\hline \multicolumn{3}{|c|}{ Number of women (out of 5 persons) } & \multicolumn{2}{|c|}{ Number of men (out of 5 persons) } \\
\hline Jacket & Trousers & Skirt & Jacket & Trousers \\
\hline $\begin{array}{l}\text { - No alterations: } 2 \\
\text { - Adjust arm length } \\
(2 \mathrm{~cm}): 3\end{array}$ & $\begin{array}{l}\text { - No alterations: } \mathbf{2} \\
\text { - Adjust waist }(2 \mathrm{~cm}) \\
\text { and hip }(2 \mathrm{~cm}): \mathbf{2} \\
\text { - Adjust length: } \mathbf{1}\end{array}$ & $\begin{array}{l}\text { - No alterations: } \mathbf{2} \\
\text { - Adjust waist }(2-4 \mathrm{~cm}) \\
\text { and hip }(2-4 \mathrm{~cm}): \mathbf{2} \\
\text { - Adjust length }(2 \mathrm{~cm}): \mathbf{1}\end{array}$ & $\begin{array}{l}\text { - No alterations: } 2 \\
\text { - Adjust arm length } \\
(2-5 \mathrm{~cm}): 3\end{array}$ & $\begin{array}{l}\text { - No alterations: } 3 \\
\text { - Adjust waist }(6-8 \mathrm{~cm}) \\
\text { and length }(3-5 \mathrm{~cm}) \\
2\end{array}$ \\
\hline
\end{tabular}




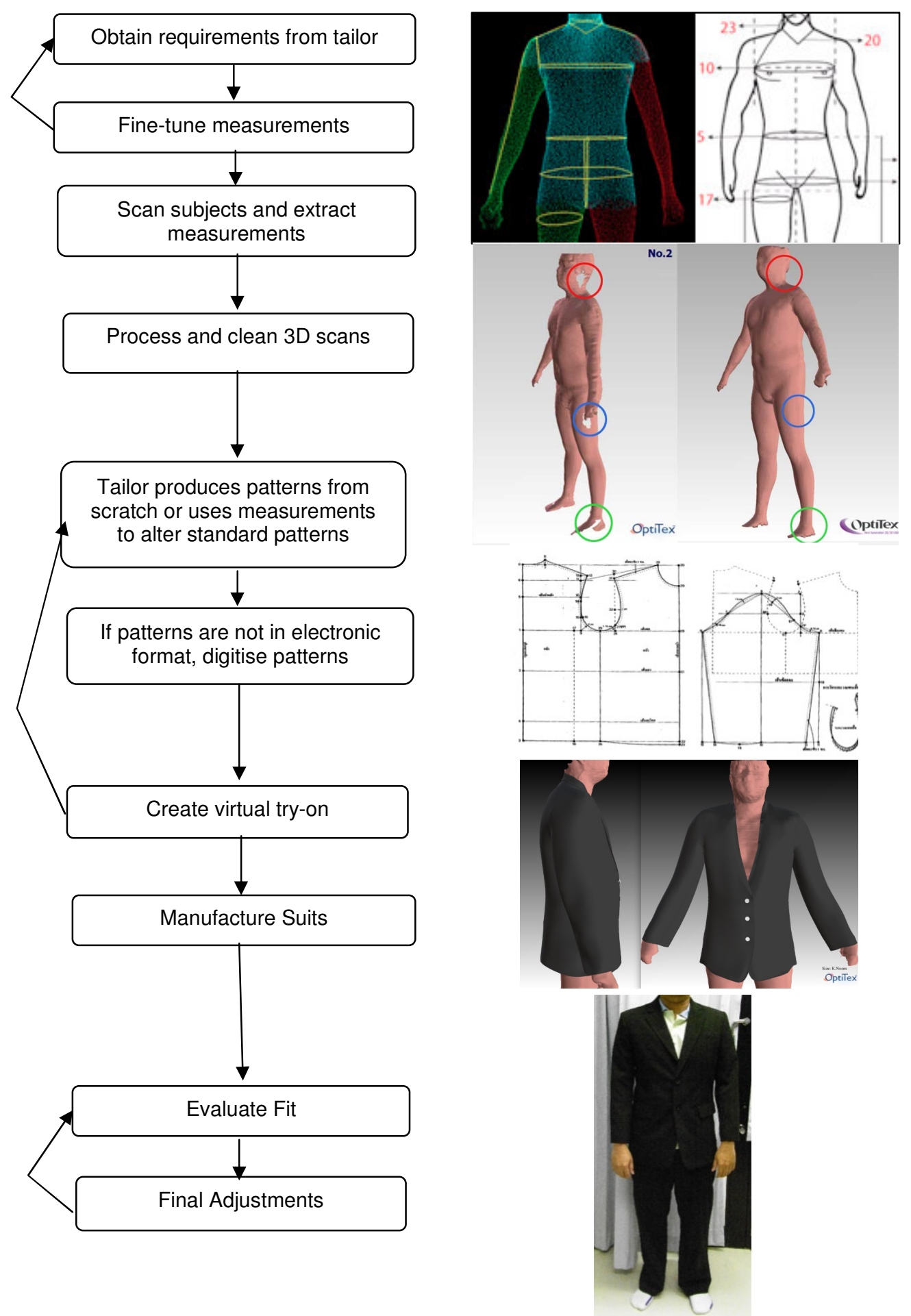

Fig. 1: MTM process being trialed in Thailand. 


\subsection{SizeThailand e-Health: Personalised Health and Food Recommendation System}

As mentioned in the background section, the aim of this project is to help reduce obesity in Thailand by developing an intelligent tool which allows the subjects taking part to monitor their body shape, clinical data, body composition monitor results and overall health online. Periodically, these data are sent to doctors to screen for obesity and other obesity-related health problems at earlier stages. The subjects also receive personalised health feedback, together with eating recommendations and guidance and on how to control their weight accordingly. This project has been running for the past 2 years with nearly 400 subjects taking part.

The progress of this project has been incremental. The first version of the system, released in 2008 , had the abilities to track changes in the body size and shape over time. It also offered visualization and statistical analysis of changes in the body shape, size and body mass index (BMI). Additionally, the system was able to predict whether certain body parts are likely to get bigger or smaller. Note that pointcloud images obtained from the 3D body scanners had to be pre-processed and compressed to make them suitable for viewing over the Internet, see Fig. 2 below.

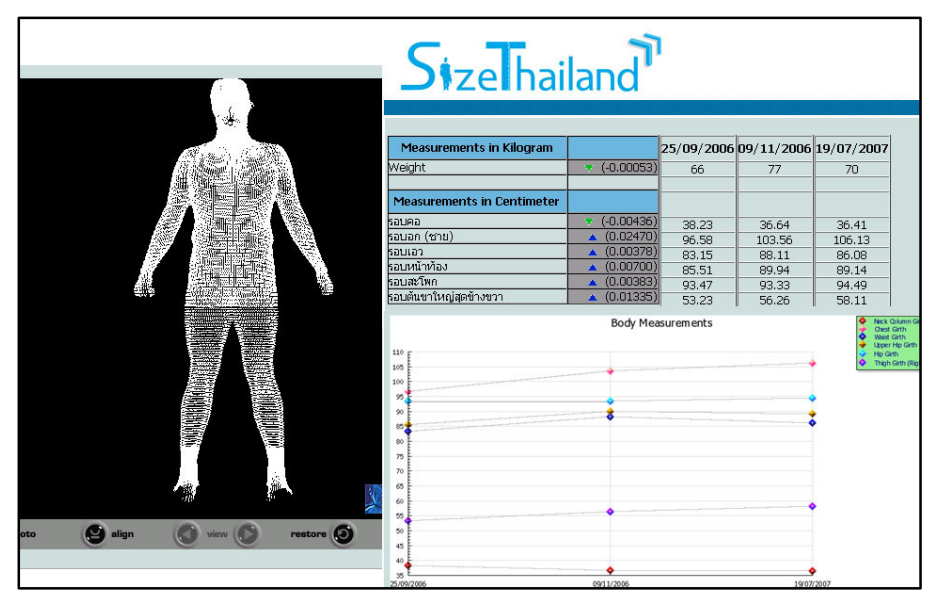

Fig. 2: The first version: personalized body size and shape monitoring tool, released in 2008.

The second release came out during the National Science and Technology Development Agency (NSTDA) staff annual medical check up in May 2009. In addition to the basic registration process, the subjects were also asked to fill in an online questionnaire on medical history and eating habits. The data collection process for this phase was re-engineered such that most tasks were carried out automatically. These included a 3D data filtering tool which automatically processes the 3D data, checking for errors that may have occurred during scanning and deciding whether there is a need to re-scan. A data uploading tool was also developed to upload 3D scans onto the server and make 2D snapshots for users with slow internet connection. This allows the subjects taking part to then access and view their data via the web straight away. Furthermore, a comparison between two scans was also added, see fig. 3, and more importantly, the summary page for each subject was created for doctors and nutritionists, allowing them to view summarized data and send personalized health feedback as shown in fig. 4. Full details of this version and its capabilities are described in [1]. 


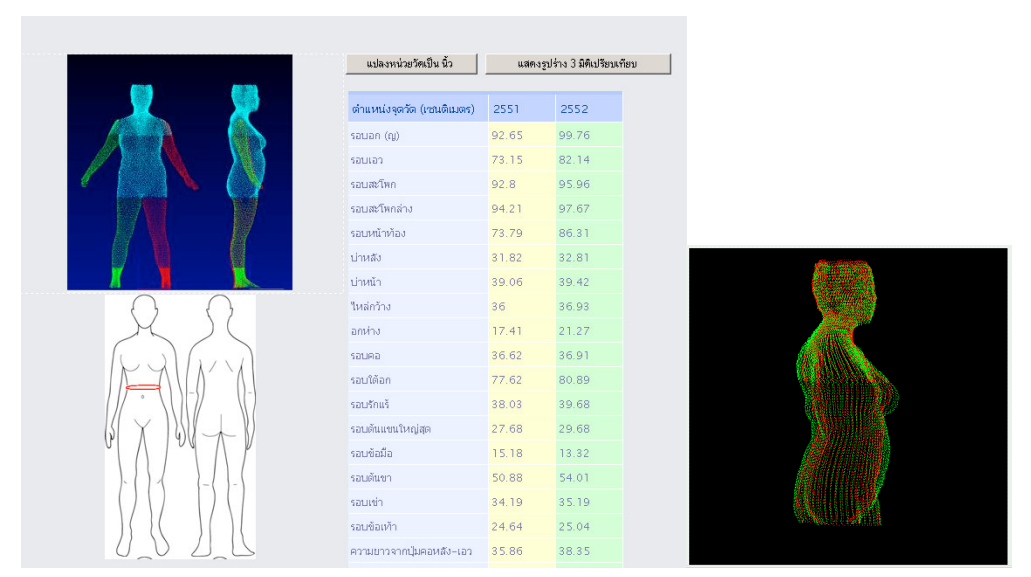

Fig. 3: Body data with key measurements (left) and comparison of two scans (right).

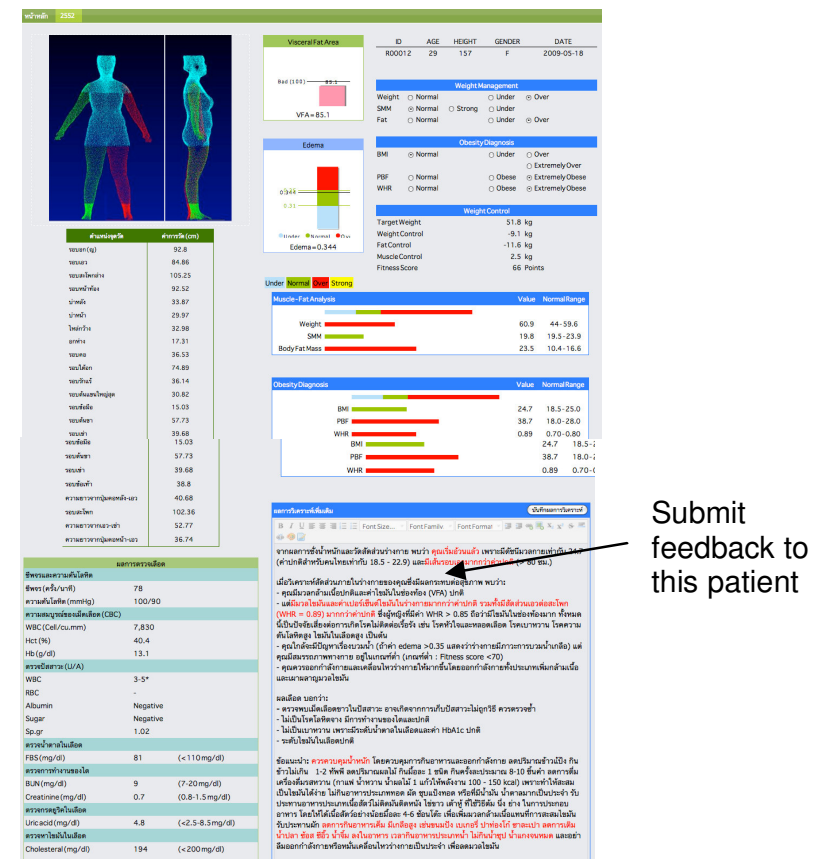

Fig. 4: Summarized view for doctors and nutritionists.

Numerous features have been added to create the latest version of the system which was released earlier this year. The additional features include different 3D display options, easy-to-view comparison of 2 scans and a personalised data summary page which dynamically compares key body measurements of the subject to those of the persons with the same age and gender, taken from the SizeThailand database, see fig. 5 . The current system is now capable of automatically generating obesity related health diagnosis. Though, these still need to be confirmed by doctors and nutritionists before they are sent back to the subjects. 

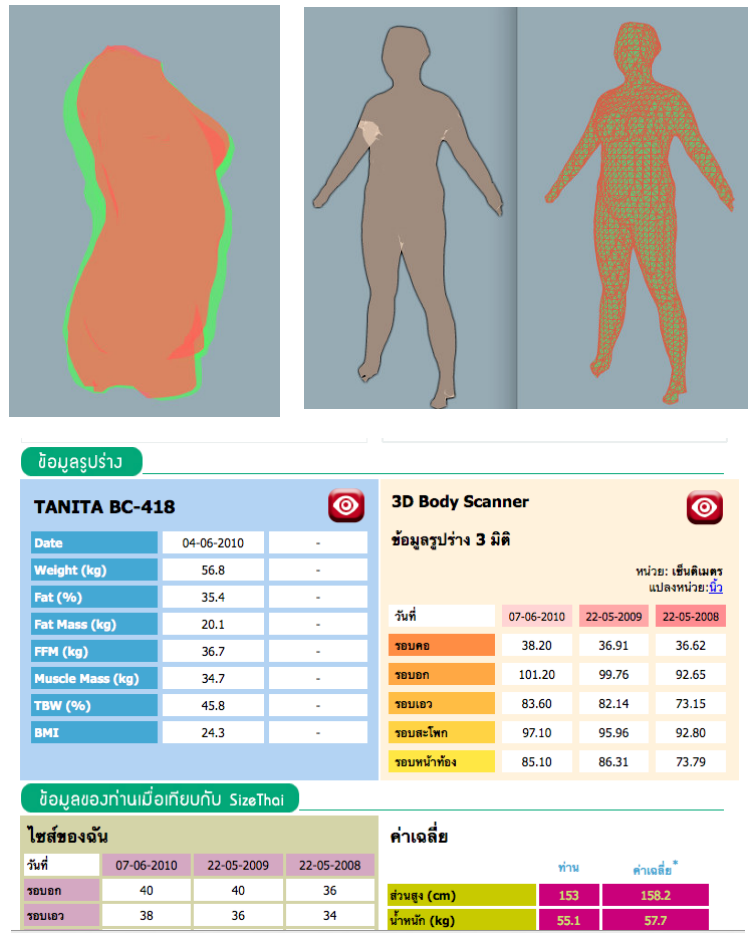
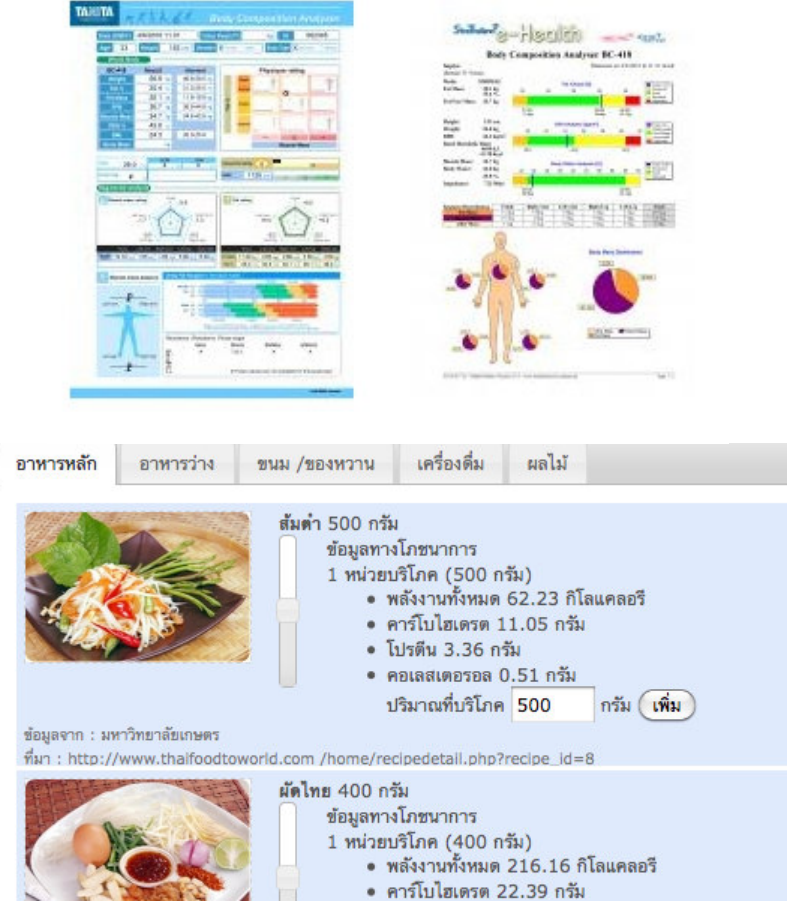

Fig. 5: Latest features which our current system offers.

Currently, we are working on incorporating food logs into the system. State-of-the-art Artificial Intelligence algorithms will be employed to give personalised food recommendations according to each subject's preferences e.g. his/her health, target body shape. Finally, there will also be a mining tool for discovering knowledge out of this continuously growing health and lifestyle database which is the main objective for this research.

\section{Conclusion}

This paper described two case studies where 3D body scanners were employed in the apparel and healthcare industries in Thailand. First, the Made-To-Measure Clothing project whose aim was to come up with a new MTM process that works best for Thai tailors. A number of suits and uniforms trials were carried out with tailors, manufacturers and fashion students. The success of a trial was measured by the percentage of the suits being well fitted, meaning that little or no alterations were required after the suits were manufactured. Summarised results of a uniform trial were presented and shown to be satisfactory. The second case study was the SizeThailand e-Health: Personalised Health and Food Recommendation System. This project aimed to help reduce obesity in Thailand by developing an intelligent tool that allowed the subjects taking part to monitor their body shape, clinical data, body composition monitor results and overall health online. The project progress was incremental and the evolution of the e-Health system was discussed. With nearly 400 subjects taking part over the past 2 years, this project has shown that 3D body scanning in healthcare is an application that people are interested in and should be pursued. 


\section{Acknowledgements}

The authors wish to thank Professor Philip Treleaven for his continual support and valuable advice throughout. We would also like to thank APP Marketing Co. Ltd. for their work on paper pattern digitization and Dr. Sriwatana Songchitsomboon from Ramathibodi hospital for her input on the SizeThailand e-Health system.

\section{References}

1. Charoensiriwath, S., (2008): "A Real-time Data Monitoring and Management System for Thailand's First National Sizing Survey", in Proceedings of the Portland International Conference on Management of Engineering \& Technology 2008 (PICTMET 2008), pp. 856-863. South Africa.

2. Department of Health, Ministry of Public Health. (2005): "Thailand Nutrition Survey 2003-2004".

3. Charoensiriwath, S., (2010): "SizeThailand e-Health: a Personalised Health Monitoring and Diagnosis System using 3D Body Scanning Technology", in Proceedings of the Portland International Conference on Management of Engineering \& Technology 2010 (PICTMET 2010), Thailand.

4. D’Apuzzo, N., (2007): "3D body scanning technology for fashion and apparel industry" in Proceedings of SPIE-IS\&T Electronic Imaging, SPIE Vol. 6491, San Jose (CA), USA.

5. Treleaven, P. and Wells, J., (2007): "3D Body Scanning and Healthcare Applications" in IEEE Computer Society, Vol. 40, No. 7, pp. 28-34.

6. Bodymetrics Made-To-Measure Jeans (accessed 2010): (http://www.bodymetrics.com/)

7. Brooks Brothers Digital Tailoring (accessed 2010): http://www.brooksbrothers.com.

8. ImageTwin Avatar Weight Loss and Body Fat Simulation Tool (accessed 2010): http://www.imagetwin.com/health fitness.html.

9. Digital Body Service (accessed 2010):http://digitalbodyshape.co.uk 